\title{
Belief Revision and Verisimilitude Based on Preference and Truth Orderings
}

\author{
Gerard R. Renardel de Lavalette $\cdot$ Sjoerd D. Zwart
}

Received: 29 April 2010/Accepted: 16 November 2010/Published online: 28 June 2011

(C) The Author(s) 2011. This article is published with open access at Springerlink.com

\begin{abstract}
In this rather technical paper we establish a useful combination of belief revision and verisimilitude according to which better theories provide better predictions, and revising with more verisimilar data results in theories that are closer to the truth. Moreover, this paper presents two alternative definitions of refined verisimilitude, which are more perspicuous than the algebraic version used in previous publications.
\end{abstract}

\section{Introduction}

In this paper we develop a formal framework that unifies Darwiche and Pearl's iterated belief revision (Darwiche and Pearl 1997) and refined verisimilitude as defined by Zwart (2001). Both belief revision and verisimilitude profit from the established unification. On the one hand, the unification provides for an answer to the epistemic problem of refined verisimilitude; on the other hand, it shows that belief revision behaves properly under the addition of truth, in the following sense.

G. R. Renardel de Lavalette ( $\bowtie)$

Johann Bernoulli Institute of Mathematics and Computer Science, Groningen University, P.O. Box 407, 9700 AK Groningen, The Netherlands

e-mail: g.r.renardel.de.lavalette@rug.nl

\section{S. D. Zwart}

Section of Philosophy, Faculty of TPM, Delft University of Technology,

P.O. Box 5015, 2600 GA Delft, The Netherlands

e-mail: s.d.zwart@tudelft.nl; s.d.zwart@tue.nl

\section{S. D. Zwart}

Subdepartment Philosophy \& Ethics, Faculty of IE \& IS, Eindhoven University of Technology, P.O. Box 513, 5600 MB Eindhoven, The Netherlands 
Revising false information of knowledge bases with true information leads to more verisimilar knowledge basis. It even turns out that revising with better information leads to more verisimilar theories.

To our knowledge until today no attempt to combine Belief Revision and Verisimilitude has been successful in the way demanded by Laudan. Laudan (1981, p. 32) rightly observes:

None of the proponents of realism has yet articulated a coherent account of approximate truth which entails that approximately true theories will, across the range where we can test them, be successful predictors.

In this paper we show that a formal framework in terms of preference and similarity relations on possible worlds helps to establish a coherent answer to Laudan's challenge. According to our framework, more verisimilar theories provide for more successful, i.e. more verisimilar, predictions and explanations. This 'downward path' from more general theories towards concrete predictions had been established already by the definition of refined verisimilitude given in Zwart (2001). Moreover, in the present framework, revising with even possibly false, but more verisimilar observations ends up with more verisimilar theories, provided that the preference relation regarding other possible empirical evidence is similar to the real verisimilitude order of this evidence. This version of the 'upward path' going from the concrete towards the more general theory, does not provide a certain method to come closer to the truth, as we are not familiar with the verisimilitude order of possible empirical evidence. It does, however, formulate and satisfy a welcome condition for a fruitful combination of verisimilitude and belief revision.

The formal framework used to establish our results is built on finite Boolean algebras (i.e. Lindenbaum algebras of propositional logic), the atoms of which correspond to models (possible worlds). We consider linear preorders on the collection of these worlds. They play the role of preference relations in the definition of (iterated) belief revision in the AGM style (Sect. 5). In the definition of (refined) verisimilitude in Sect. 6, we shall use these linear preorders as similarity functions.

The rest of the paper is structured as follows. In the next section we will first introduce the main ideas behind research into belief revision and verisimilitude. Moreover, we describe an example of Sven Ove Hansson which nicely illustrates the intentions of our exercise. We will return to this example in Sect. 7 to illustrate the mechanisms of our framework. Section 3 is mainly dedicated to the introduction of propositional logic in the form of Boolean algebra, the basic framework of our work. In Sect. 4 we introduce the main formal apparatus of the paper: preferences and preference orders (a dual of epistemic entrenchment). In the subsequent Sects. 5 and 6 we show how (iterated) belief revision and (refined) verisimilitude are defined in our framework. Especially, the new definitions of refined verisimilitude as previously defined in Zwart (2001) turn out to be useful. Finally in the penultimate Sect. 7, we show how neatly existing belief revision and refined verisimilitude fit together and successfully fulfill Laudan's challenge. We end in Sect. 8 with the conclusions and describe prospects of future research. 


\section{Informal Exposition of Verisimilitude and Belief Revision}

Investigations into verisimilitude started within the school of scientific realism in philosophy of science. In 1963, Popper proposed a formal definition of the idea that a given (possibly false) theory can be more similar to "the true theory" than another, competing (possibly false) theory. The research project of verisimilitude really got off the ground only when it was discovered that Popper's definition in fact failed to compare any two nonequivalent false theories - which had precisely been the main aim of the definition.

The main subject of the verisimilitude project is formulated in relation to a formal language that is assumed to include a (usually) complete empirical truth $\tau$. If $\tau$ is complete, any synthetic sentence is either a consequence of $\tau$, or implies $\neg \tau$. Within the context of classical, non-modal logic, empirical incompleteness of $\tau$ implies that the underlying language comprises non-referring propositional variables. Given a language, verisimilitude investigations concern two questions. The first question reads: How are we to define the similarity between an arbitrary theory in the language and its true theory $\tau$ ? Answering this question about the definition of verisimilitude, we may assume to be familiar with $\tau$. The second question about verisimilitude reads: when confronted with two different (scientific) theories, how are we to find out which of the two theories is more verisimilar or closer to the truth $\tau$ ? This epistemic question of verisimilitude is the more practical one. Obviously, when we formulate the answer to the epistemic question, we may not assume that we know the true theory $\tau$. When we have to decide whether the mechanics of Descartes rather than that of Newton is more verisimilar we do not know the full truth, although, perhaps, we know some elements in it, e.g. some very reliable observations made by different researchers. Until today, no generally accepted solution to the epistemic problem of verisimilitude exists. In the present paper we will show that belief revision provides an appropriate answer to the epistemic question of the refined verisimilitude.

The study of belief revision is rooted in the holistic or coherentist tradition of Duhem (1954) and Quine and Ullian (1978). Its main objective has been to answer the question how to revise an existing body of knowledge $K$ when confronted with new evidence $\varphi$ that may contradict $K$. In Alchourrón et al. (1985), formulated postulates that turned out to be an important part of the answer to the revision question. The AGM-postulates put constraints on how to revise $K$ into a new knowledge set $K * \varphi$, but do not uniquely determine one revision operator. Alchourrón et al. (1985, p. 88) claim that their postulates

... exhaust what can be said about revisions and contraction in logical and settheoretical terms only. This means that we must seek further information about the epistemic status of the elements of a knowledge state to solve the uniqueness problem.

The problem with the AGM postulates is that they only provide one-step revisions. After a revision of $K$ by $\varphi$ has taken place, the AGM-postulates fail to give indications how one should come to a new entrenchment relation $\leqslant_{K * \varphi}$. They fail 
therefore to indicate how to execute a next revision of $K * \varphi$ by $\psi$. This drawback of the AGM approach was readily remarked and in 1954, Darwiche and Pearl added four postulates for iterated revisions.

For our purposes iterated revisions are unavoidable. The gist of combination of belief revision and verisimilitude is the answer to the question how theories behave under revisions on the long run, and then one-step revisions are insufficient. What we want is to show that iterated truthful revisions of theories far from the truth eventually end up with theories that are much closer to the truth.

For the purposes mentioned, we prefer to use the formulation of belief revision in terms of preferences as presented by Grove (1988). This approach is dual to entrenchment: $\varphi$ is preferred to $\psi(\varphi<\psi)$ iff $\neg \psi$ is better entrenched than $\neg \varphi\left(\neg \varphi<\mathrm{e}^{\mathrm{e}} \neg \psi\right)$.

Let us consider a simple example, due to Sven Ove Hansson (private conversation), to illustrate how the result of belief revision depends on the preference.

Example 1 (Hansson) Let the present body of knowledge $\kappa$ be $P_{0} \wedge \neg P_{1}$. How are we to revise $\kappa$ when confronted with new evidence $\varphi=P_{0} \leftrightarrow P_{1}$ ? Here the idea of preference (or its dual, epistemic entrenchment) comes in. If (i) $\neg P_{0} \wedge \neg P_{1}$ is preferred to $P_{0} \wedge P_{1}$, i.e. $\neg P_{0} \wedge \neg P_{1}<P_{0} \wedge P_{1}$, then $\kappa * \varphi=\neg P_{0} \wedge \neg P_{1}$; but if (ii) $P_{0} \wedge P_{1}<\neg P_{0} \wedge \neg P_{1}$ then $\kappa * \varphi=P_{0} \wedge P_{1}$. And if (iii) $P_{0} \wedge P_{1}$ and $\neg P_{0} \wedge \neg P_{1}$ are equally preferred, then $\kappa * \varphi=P_{0} \leftrightarrow P_{1}$. Now if the truth is supposed to be $P_{0} \wedge$ $P_{1}$, then revision (ii) is better than (i), since it leads us closer to the truth.

The example concerns only a one-step revision, but is readily expanded to iterated revisions. To handle iterated revisions, one should indicate how existing preference relations should be updated after a revision. It turned out that the most practical way to formulate the combination of iterated revisions and verisimilitude was in terms of preference relations. The reason of cause being that iterated revisions and verisimilitude all come down to the comparison of orders and the revisions of orders into new ones.

\section{Formal Preliminaries}

As our logical basis, we take classical (two-valued) propositional logic over a finite collection of propositional variables $\mathrm{PVAR}=\left\{P_{0}, \ldots, P_{n-1}\right\}$. On the collection FORM of formulae, we have the usual entailment relation $\vdash$ and the logical equivalence relation $\equiv$. In the sequel, language $L$ refers to the triple $\langle F O R M, \vdash, \equiv\rangle$.

As is well known, FORM is (modulo logical equivalence) isomorphic to the Boolean algebra $\mathrm{BA}(n)$ over $n$ generators. Recall that $\mathrm{BA}(n)$ is finite but large, having $2^{2^{n}}$ elements. Since FORM is finite (modulo logical equivalence), every knowledge base or theory, i.e. every collection of formulae, is equivalent with a single formula: the conjunction of its (finitely many non-equivalent) elements. We will use this property throughout this paper, representing theories cq. knowledge bases by single formulae. 
The collection ATOM of atoms of $\mathrm{BA}(n)$ contains the $2^{n}$ formulae of the form $(\neg) P_{0} \wedge \ldots \wedge(\neg) P_{n-1}$ : they are the minimal elements of $\operatorname{BA}(n)-\{\perp\}$. (NB: observe that, in general, an atom is not an atomic formula!) Atoms are interesting formulae, as they can be seen as models for propositional logic: $\varphi$ holds in the model corresponding to atom $\alpha$ iff $\alpha \vdash \varphi$. As is well known, every formula in $\operatorname{BA}(n)$ can be written uniquely as a disjunction of atoms: $\varphi=\bigvee$ atom $(\varphi)$, where atom $(\varphi)=\{\alpha \in \operatorname{ATOM} \mid \varphi \vdash \alpha\}$. Some properties of atoms are:

$$
\begin{aligned}
& \varphi \vdash \psi \text { iff atom }(\varphi) \subseteq \text { atom }(\psi) \\
& \text { atom }(\top)=\operatorname{ATOM} \\
& \operatorname{atom}(\perp)=\emptyset \\
& \operatorname{atom}(\neg \varphi)=\operatorname{ATOM}-\operatorname{atom}(\varphi) \\
& \text { atom }(\varphi \wedge \psi)=\operatorname{atom}(\varphi) \cap \operatorname{atom}(\psi) \\
& \text { atom }(\varphi \vee \psi)=\operatorname{atom}(\varphi) \cup \operatorname{atom}(\psi)
\end{aligned}
$$

\section{Preferences}

Some situations are preferred to others: this simple consideration is the basis for preferences. We identify situations with models, hence with atoms, so preference can be modeled as an order relation $\leqslant$ on atoms. ${ }^{1}$

The preference orders considered in this paper are total preorders on ATOM, i.e. they are reflexive, transitive and connected:

$$
\begin{aligned}
& \forall \alpha(\alpha \leqslant \alpha) \\
& \forall \alpha \beta \gamma(\alpha \leqslant \beta \& \beta \leqslant \gamma \Rightarrow \alpha \leqslant \gamma) \\
& \forall \alpha \beta(\alpha \leqslant \beta \text { or } \alpha=\beta \text { or } \beta \leqslant \alpha)
\end{aligned}
$$

Given a preference order $\leqslant$, the strict preference order $<$ is defined by $\alpha<\beta$ iff $\beta \leqslant \alpha$, and the preference equivalence $\cong$ by $\alpha \cong \beta$ iff $\alpha \leqslant \beta \& \beta \leqslant \alpha$.

Preference orders on a finite domain can be represented by preference functions $p:$ ATOM $\rightarrow \mathbb{N}$. The idea is that $p(\alpha)$ indicates the degree of preference of $\alpha: \alpha<_{p}$ $\beta$, i.e. $p$ prefers $\alpha$ to $\beta$, whenever $p(\alpha)<p(\beta)$. Conversely, the preference function $p \leqslant$ of a preference order $\leqslant$ can be defined by

$$
p_{\leqslant}(\alpha)=\max \left\{k \mid k=0 \text { or } \exists \beta_{1}, \ldots, \beta_{k}\left(\beta_{1}<\cdots<\beta_{k}<\alpha\right)\right\}
$$

The collection of preference functions is called PREF. Different preference functions may lead to the same preference order, e.g. $p$ and $\lambda \alpha . p(\alpha)+1$. There is, however, a one-to-one correspondence between preference orders and normal preference functions $p \in$ NPREF that have an initial segment $[0, n]$ as their range. Every preference function can be normalized by the mapping norm, defined by

\footnotetext{
1 Of course, we are well aware of the examples of Miller (1974) showing that preferences of models are not absolute, but depend on the choice of atomic propositions used to identify the models or possible worlds. For more details the reader is referred to Zwart (2001, Chap. 5).
} 


$$
\operatorname{norm}(p)(\alpha)=\#\{k \mid \exists \beta \in \operatorname{ATOM} p(\beta)=k<p(\alpha)\}
$$

where $\# X$ denotes the number of elements of $X$. So a preference order and its corresponding normal preference function are two sides of the same coin, and we shall use these two representations interchangeably in this paper.

A preference function $p$ on atoms can be extended to a mapping $p:$ FORM $\rightarrow \mathbb{N}$ on all formulae by

$$
\begin{aligned}
& p(\varphi)=\min \{p(\alpha) \mid \alpha \in \operatorname{atom}(\varphi)\} \quad \text { if } \varphi \not \equiv \perp \\
& p(\perp)=\max (p)+1
\end{aligned}
$$

where $\max (p)=\max \{p(\alpha) \mid \alpha \in \operatorname{ATOM}\}$ (and similarly for $\min (p)$, to be used later). A preference order $\leqslant$ on atoms is extended to formulae by

$$
\varphi \leqslant \psi \text { iff } \forall \alpha \in \operatorname{atom}(\psi) \exists \beta \in \operatorname{atom}(\varphi) \beta \leqslant \alpha
$$

alternatively, it can be obtained from $p:$ FORM $\rightarrow \mathbb{N}$ by $\varphi \leqslant p \psi \Leftrightarrow p(\varphi) \leqslant p(\psi)$. A preference order on FORM has the following properties:

$\leqslant$ is a total preorder;

$\leqslant$ subsumes $\dashv$ (the inverse of $\vdash$ ): $\varphi \vdash \psi$ implies $\psi \leqslant \varphi$;

$\leqslant$ is disjunctive: $\varphi \leqslant \varphi \vee \psi$ or $\psi \leqslant \varphi \vee \psi$;

$\leqslant$-maximal formulae are inconsistent: if $\psi \leqslant \varphi$ for all $\psi$, then $\varphi \vdash \perp$.

The disjunctivity property of $\leqslant$ is a consequence of the use of the minimum operator in the definition of $p(\varphi)$. It is not hard to see that every relation on formulae that satisfies these properties is fully determined by its behaviour on atoms.

A formula $\varphi$ can also be seen as a preference: atoms in atom $(\varphi)$ are preferred to atoms not in atom $(\varphi)$. Going in the other direction, we can reduce a preference to a formula consisting of the most preferred atoms. So we define form : PREF $\rightarrow$ FORM and pref : FORM $\rightarrow$ PREF by

$$
\begin{aligned}
& \operatorname{form}(p)=\bigvee\{\alpha \mid p(\alpha)=\min (p)\} \\
& \operatorname{pref}(\varphi)=\lambda \alpha .(\text { if } \alpha \vdash \varphi \text { then } 0 \text { else 1) }
\end{aligned}
$$

So $\operatorname{pref}(\varphi)(\alpha)=0$ if $\alpha \vdash \varphi$, and $\operatorname{pref}(\varphi)(\alpha)=1$ if $\alpha \nvdash \varphi$. We have that form and pref are antimonotonic in the following sense:

$$
\begin{aligned}
p \leqslant q & \Rightarrow \operatorname{form}(q) \vdash \operatorname{form}(p) \quad(p, q \in \mathrm{NPREF}) \\
\varphi \vdash \psi & \Rightarrow \operatorname{pref}(\psi) \leqslant \operatorname{pref}(\varphi)
\end{aligned}
$$

Now that we know what preferences are, the question remains how we may obtain them. One way to obtain a preference is via a distance function $d: \mathrm{ATOM}^{2} \rightarrow \mathbb{N}$ satisfying

$$
\begin{array}{ll}
d(\alpha, \beta)=0 \text { iff } \alpha=\beta & \text { (identity) } \\
d(\alpha, \beta)=d(\beta, \alpha) & \text { (symmetry) } \\
d(\alpha, \beta)+d(\beta, \gamma) \leqslant d(\alpha, \gamma) & \text { (triangle inequality) }
\end{array}
$$

Such a distance function can be generalized to all formulae by 


$$
d(\varphi, \psi)=\min \{d(\alpha, \beta) \mid \alpha \in \operatorname{atom}(\varphi), \quad \beta \in \operatorname{atom}(\psi)\}
$$

observe that this generalization $d: \mathrm{FORM}^{2} \rightarrow \mathbb{N}$ is only a weak distance function, satisfying symmetry and the triangle inequality but not the identity property.

Given a weak distance function $d$ defined on formulae and a formula $\varphi$, we can define a preference function $\operatorname{pref}(d, \varphi)$ :

$$
\operatorname{pref}(d, \varphi)(\alpha)=d(\alpha, \varphi)
$$

so the degree of preference of $\alpha$ corresponds with the distance from $\varphi$. We have

$$
\varphi \vdash \psi \Rightarrow \operatorname{pref}(d, \varphi) \geqslant \operatorname{pref}(d, \psi)
$$

Observe that the unary function pref defined earlier is a special case of the binary pref, based on the trivial distance function $d$ defined by $d(\alpha, \alpha)=0$ and $d(\alpha, \beta)=1$ if $\alpha \neq \beta$.

A particular example of a distance function on atoms is the Hamming distance $d_{H}$, which counts the number of propositional variables on which the two atoms differ:

$$
d_{H}(\alpha, \beta)=\#\{P \in \mathrm{PVAR} \mid \alpha \vdash P \text { xor } \beta \vdash P\}
$$

We shall use this distance function when discussing verisimilitude.

\section{Belief Revision}

Traditionally (see Alchourrón et al. (1985)), belief revision is defined in terms of an epistemic entrenchment order $\leqslant^{e}$. The idea is that the degree of epistemic entrenchment determines which of two beliefs $\varphi$ and $\psi$ has to be given up when their combination has become untenable: in a situation where $\varphi \wedge \psi$ is inconsistent and $\varphi<{ }^{\mathrm{e}} \psi$ (i.e. $\psi$ is more entrenched that $\varphi$ ), it is preferred to give up $\varphi$ and retain $\psi$. The knowledge set $K$ associated with $\leqslant^{\mathrm{e}}$ is defined as the collection of the non$\leqslant$-minimal formulae.

In the case of a finite logic, $\leqslant^{e}$ can be defined straightforwardly in terms of the dual atoms (i.e. negations of atoms). This approach has been dualized by Grove (1988), replacing dual atoms by atoms, and $\leqslant^{\mathrm{e}}$ by its dual: a preference order $\leqslant$ as described in Sect. 4 that satisfies $\varphi \leqslant^{\mathrm{e}} \psi$ iff $\neg \varphi \leqslant \neg \psi$. Since the use of atoms is (in our eyes) more intuitive than dual atoms, we adopt Grove's representation of belief revision based on preference order and preference functions. Since our logic is finite, the knowledge set $K$ can be replaced by its conjunction $\kappa=\bigwedge K$. When $\leqslant$ equals $\leqslant_{p}$, the preference order generated by preference function $p$, then the conjunction $\kappa=\kappa_{p}$ of the knowledge set associated with $\leqslant_{p}$ equals form $(p)$, the disjunction of the most preferred atoms (i.e. with minimal $p$-value).

The theory of belief revision usually proceeds by defining belief revision as an operation on formulae: $\kappa * \varphi$ is the revision of $\kappa$ with evidence $\varphi$. When working with preferences, the revision of $\kappa=\kappa_{p}=$ form $(p)$ with $\varphi$ can be defined as the disjunction of all atoms in atom $(\varphi)$ that are most preferred by $p$ : 


$$
\kappa_{p} * \varphi=\bigvee\{\alpha \mid \alpha \vdash \varphi \& p(\alpha)=p(\varphi)\}
$$

(recall that $p(\varphi)$ is defined as the minimum of the preference of the atoms in atom $(\varphi))$. When $\kappa_{p}$ and $\varphi$ are consistent, atom $\left(\kappa_{p}\right)$ and atom $(\varphi)$ have one or more atoms $\alpha$ in common: they satisfy $p(\alpha)=0$ and $\alpha \vdash \varphi$, so $p(\varphi)=\min \{p(\alpha) \mid \alpha \vdash$ $\varphi\}=0$. In this case we have a conservative revision $\kappa_{p} * \varphi=\kappa_{p} \wedge \varphi$. However, when $\kappa_{p} \wedge \varphi \vdash \perp$, we have $\kappa_{p} * \varphi=\bigvee\{\alpha \mid \alpha \vdash \varphi \& p(\alpha)=k\}$ for some $k>0$, which we call a Popperian revision.

Observe that, in (1), the revision operator $*$ is in fact an operation on a preference $p$ and a formula $\varphi$, although the notation $\kappa_{p} * \varphi$ suggest that it is a binary operation on formulae. As a consequence, belief revision in this form cannot be iterated: for the proper definition of $\left(\kappa_{p} * \varphi\right) * \psi$, the preference function associated with $\kappa_{p} * \varphi$ is required.

To deal with this, we shall generalize (1) in two steps. The first is to lift revision of formula $\kappa$ with formula $\varphi$ as defined in (1) to a revision of preference $p$ with $\varphi$. This leads to the preference $p * \varphi$, which should satisfy $\kappa_{p * \varphi}=\kappa_{p} * \varphi$. Now iterated belief revision $(p * \varphi) * \psi$ is possible. This idea is pursued by Darwiche and Pearl (1997), where the following postulates on iterated revision are put forward:

$$
\begin{aligned}
\varphi \vdash \psi & \Rightarrow(p * \psi) * \varphi=p * \varphi \\
\varphi \vdash \neg \psi & \Rightarrow(p * \psi) * \varphi=p * \varphi \\
K_{p * \varphi} \vdash \psi & \Rightarrow K_{(p * \psi) * \varphi} \vdash \psi \\
K_{p * \varphi} \forall \neg \psi & \Rightarrow K_{(p * \psi) * \varphi} \forall \neg \psi
\end{aligned}
$$

We go one step further, and lift revision to a revision operator $*:$ PREF $^{2} \rightarrow$ PREF on preference functions. A related approach is described by Nayak (1994), where the revision of an entrenchment order with another entrenchment order is defined. We consider some candidates for the definition of preference revision:

$$
\begin{aligned}
\left(p *_{1} q\right)(\alpha) & =p(\alpha)+(\max (p)+1) \cdot q(\alpha) \\
\left(p *_{2} q\right)(\alpha) & =0 \quad \text { if } q(\alpha)=0 \& p(\alpha)=p(\text { form }(q)) \\
& =p(\alpha)+1 \quad \text { elsewhere } \\
\left(p *_{3} q\right)(\alpha) & =0 \quad \text { if } q(\alpha)=0 \& p(\alpha)=p(\operatorname{form}(q)) \\
& =q(\alpha) \quad \text { if } q(\alpha)>0 \& p(\alpha)=0 \\
& =p(\alpha) \quad \text { elsewhere } \\
\left(p *_{4} q\right)(\alpha) & =p(\alpha)-p(\text { form }(q)) \quad \text { if } q(\alpha)=0 \\
& =p(\alpha)+q(\alpha) \quad \text { elsewhere }
\end{aligned}
$$

For the first two candidates, there is an alternative definition possible in terms of preference orders: 


$$
\begin{array}{ll}
\alpha \leqslant_{p *_{1} q} \beta & \text { iff } \quad \alpha<_{q} \beta \text { or }\left(\alpha \cong_{q} \beta \& \alpha \leqslant_{p} \beta\right) \\
\alpha \leqslant_{p *_{2} q} \beta & \text { iff } \quad \alpha \leqslant_{p} \beta \text { or }(q(\alpha)=0 \& p(\alpha)=p(\operatorname{form}(q)))
\end{array}
$$

Observe that $\leqslant_{p *_{1} q}$ is the lexicographical combination of $\leqslant_{p}$ and $\leqslant_{q} ; p *_{2} q$ can be paraphrased as 'follow $p$ as much as possible'.

Traditional belief revision as defined in (1) can be defined in terms of preference revision by $\kappa_{p} * \varphi=$ form $(p) * \varphi=$ form $\left(p *_{i} \operatorname{pref}(\varphi)\right)(i=1,2,3,4)$, where the result does not depend on $i$ : we have in all cases $\kappa_{p} * \varphi=\bigvee\{\alpha \mid \alpha \vdash \varphi \&$ $p(\alpha)=p(\varphi)\}$.

Preference revision with a formula is obtained by $p *_{i} \varphi=p *_{i} \operatorname{pref}(\varphi)$ $(i \in\{1,2,3,4\})$. This leads to

$$
\begin{aligned}
& \left(p *_{1} \varphi\right)(\alpha)=p(\alpha) \quad \text { if } \alpha \vdash \varphi \\
& =p(\alpha)+\max (p)+1 \quad \text { if } \alpha \nvdash \varphi \\
& \left(p *_{2} \varphi\right)(\alpha)=0 \quad \text { if } \alpha \vdash \varphi \text { and } p(\alpha)=p(\varphi) \\
& =p(\alpha)+1 \text { if } \alpha \nvdash \varphi \text { or } p(\alpha) \neq p(\varphi)
\end{aligned}
$$

and similar for $*_{3}$ and $*_{4}$. In all cases, the Darwiche-Pearl postulates hold.

\section{Verisimilitude}

The starting point for verisimilitude is the strongest empirically true theory $\tau$ expressible in $L$. Normally, $\tau$ is assumed to be complete, in the sense that any contingent sentence is either a consequence of $\tau$, when it is empirically true, or implies $\neg \tau$ when it is false. To stay as general as possible, however, our formal framework does allow for the degenerated case in which the truth is incomplete and $\tau \notin$ ATOM. Note that in such cases, language $L$ is inadequate in the sense that it comprises propositional variables which nature does not verify nor falsify. After fixing $L$ and its strongest empirical truth $\tau$, we set out to obtain a relation $\leqslant$ v (and its strict version $<^{\mathrm{v}}$ ) of verisimilitude, where $\varphi<{ }^{\mathrm{v}} \psi$ expresses that $\varphi$ is more verisimilar ('closer to the truth') than $\psi$.

As for similarity between atoms, we shall obtain a verisimilitude order on FORM from a total preorder on atoms. So let some similarity function $t: A T O M \rightarrow \mathbb{N}$ be given. We assume that the $t$-minimal atoms are precisely the atoms of $\tau$, i.e. atom $(\tau)=\{\alpha \mid t(\alpha)=0\}$. A natural example of a similarity based on $\tau$ is $t=\lambda \alpha$. $d_{H}(\alpha, \tau)$ where $d_{H}$ is the Hamming distance between atoms, introduced at the end of Sect. 4. So $t(\alpha)=0$ if $\alpha \in$ atom $(\tau)$; in general, $t(\alpha)$ is the minimal number of changes in $\alpha$ (i.e. adding or removing a negation sign) required to obtain an atom in $\tau$.

Given $t$ and $\tau=\bigvee\{\alpha \mid t(\alpha)=0\}$, we formulate some requirements on the verisimilitude relation $\left(\leqslant^{\mathrm{v}}\right)=\left(\leqslant_{t}^{\mathrm{v}}\right)$ associated with $t$ :

$$
\begin{gathered}
\leqslant \vee \text { is a preorder, i.e. reflexive and transitive } \\
\varphi \vdash \psi \vee \tau \Rightarrow \varphi \leqslant \vee \psi(\text { content condition) }
\end{gathered}
$$




$$
\begin{aligned}
& t(\alpha) \leqslant t(\beta) \Rightarrow \alpha \leqslant{ }^{v} \beta \text { for atoms } \alpha, \beta \text { (likeness condition) } \\
& \leqslant \text { commutes with } \vdash \text { and with } \dashv \text {, i.e. } \\
& \left(\leqslant^{\mathrm{v}} \cdot \vdash\right)=\left(\vdash \cdot \leqslant^{\mathrm{v}}\right) \text { and }\left(\leqslant^{\mathrm{v}} \cdot \dashv\right)=\left(\dashv \cdot \leqslant^{\mathrm{v}}\right) \\
& \varphi \leqslant{ }^{\vee} \psi \Leftrightarrow \neg \psi \leqslant \vee \vee \varphi \text { (contraposition) }
\end{aligned}
$$

Here $\dashv$ is the inverse of $\vdash$, and - denotes composition of relations: $x R \cdot S y$ iff there is a $z$ with $x R z$ and $z S y$.

Requirement (2) is a rather natural requirement for a ordering relation. The content condition (3) and the likeness condition (4) have been discussed extensively in Zwart (2001), Zwart and Franssen (2007). Niiniluoto (1987, p. 233) introduces the truth content criterion M7, which comes down to the content condition (3) restricted to false sentences $\psi$. In addition, Niiniluoto introduces the similarity condition M6, being very similar to (4): if the similarity between atom $\alpha$ and the truth is larger than that of $\beta$ and the truth, the former is more verisimilar. This natural condition lies at the heart of likeness approaches, such as Niiniluoto's minsum measure in Niiniluoto (1987).

The commutation requirement (5) is related to Laudan's challenge, as quoted in the Introduction. To see this, we formulate the following consequence of (5), where $T$ and $T$ are theories (denoted as formulae):

if $T \leqslant^{v} T^{\prime}$, then (i) for every consequence $\varphi^{\prime}$ of $T^{\prime}$ there is a consequence $\varphi$ of $T$ with $\varphi \leqslant^{\mathrm{v}} \varphi^{\prime}$, and (ii) for every consequence $\varphi$ of $T$ there is a consequence $\varphi^{\prime}$ of $T^{\prime}$ with $\varphi \leqslant{ }^{v} \varphi^{\prime}$.

In slogan: the better theory has the better consequences. As (5) is a necessary condition for a successful acceptance of Laudan's challenge, it is a desirable property for verisimilitude. In our framework of a Boolean algebra, empirical predictions may be represented by the weakest nontrivial consequences of a theory, viz. its dual atoms $\delta=(\neg) P_{0} \vee \ldots(\neg) P_{n-1}$. This enables us to show that the better/ worse theory simply has the better/worse consequences, even in the sense that the better of false theories has the better dual atoms, i.e. empirical consequences. Something similar is out of reach of other content definitions of verisimilitude such as those of Miller's (1978) and Kuipers' symmetric difference definition (Kuipers 2000, p. 151): in their definitions, all false atoms (i.e. atoms not in atom $(\tau)$ ) are incomparable regarding their similarity with the truth.

Finally, let us consider contraposition (6). In Zwart and Franssen (2007), it turned out that contraposition defines an important watershed in the verisimilitude literature. It forces $\neg \tau$ to be the worst sentence if $\tau$ is the truth, such that $\tau$ and $\neg \tau$ become the lower and upper limit of all sentences in the language. By doing so, contraposition demarcates the differences between likeness and content definitions of verisimilitude, since according to likeness definitions this upper limit is $\tau^{*}$, which is the atom of the language with maximal distance from $\tau$. Consequently, contraposition (6) is a desirable property for content definitions of verisimilitude. Here, it turns out to be one of the reasons for refined verisimilitude to fit closely to the framework of belief revision. 
In a first attempt to satisfy these properties, we define two order relations. The first is an attempt to realize the content condition (3), the second aims to satisfy the likeness condition (4).

Definition 1 (content and likeness order) The content order $\sqsubseteq$ is defined by:

$$
\varphi \sqsubseteq \psi \Leftrightarrow \varphi \vdash \psi \vee \tau
$$

and the likeness order $\preccurlyeq$ by:

$$
\varphi \preccurlyeq \psi \Leftrightarrow \exists t \text {-increasing bijection } f: \text { atom }(\varphi \wedge \neg \psi) \rightarrow \text { atom }(\psi \wedge \neg \varphi)
$$

Here $t$-increasing means: if $\alpha \in \operatorname{dom}(f)$ then $t(\alpha) \leqslant t(f(\alpha))$. We call the $f$ in (7) a witness for $\varphi \preccurlyeq \psi$.

Remark The original definition of the likeness order in Zwart (2001) reads

$$
\varphi \preccurlyeq \psi \Leftrightarrow \exists t \text {-increasing bijection } g: \operatorname{atom}(\varphi) \rightarrow \operatorname{atom}(\psi)
$$

where $t$ is defined by the Hamming distance from the formula $\tau$, i.e. $t(\alpha)=d_{H}(\tau, \alpha)$. Apart from the specific choice of $t$, both definitions of the likeness order are equivalent. For if $f$ satisfies (7) then $g$ defined by

$$
\begin{array}{rlrl}
g(\alpha) & =f(\alpha) & \text { if } \alpha \in \operatorname{atom}(\varphi \wedge \neg \psi) \\
& =\alpha & & \text { if } \alpha \in \operatorname{atom}(\varphi \wedge \psi)
\end{array}
$$

satisfies (8); and if $g$ satisfies (8) then $f$, defined by

$$
\begin{gathered}
\text { if } \alpha \in \operatorname{atom}(\varphi \wedge \neg \psi), \\
\text { where } n=1+\max \left\{k \mid g^{k}(\alpha) \in \text { atom }(\varphi)\right\}
\end{gathered}
$$

satisfies (7). Here $g^{n}$ denotes $n$-times iterated application of $g: g^{1}(\alpha)=g(\alpha), g^{2}(\alpha)=$ $g(g(\alpha))$, etc. In the sequel, we shall use (7) or (8) as the definition of $\preccurlyeq$, whatever suits best.

Lemma 1 (properties of $\sqsubseteq$ and $\preccurlyeq) \sqsubseteq$ and $\preccurlyeq$ are preorders, satisfy contraposition, and commute with $\vdash$ and $\dashv$. Moreover, $\sqsubseteq$ satisfies the content condition and $\preccurlyeq$ the likeness condition, but not the other way round.

Proof See the Appendix.

So neither $\sqsubseteq$ nor $\preccurlyeq$ satisfies all requirements (2-6). Let us try to combine both candidates. The next lemma says that the order of composition is irrelevant.

Lemma 2 (commuting relations) $\sqsubseteq$ and $\preccurlyeq$ commute, i.e. $(\sqsubseteq \cdot \preccurlyeq)=(\preccurlyeq \cdot \sqsubseteq)$.

Proof See the Appendix.

Lemma 2 enables us to combine $\sqsubseteq$ and $\preccurlyeq$ into a stronger overarching verisimilitude concept.

Definition 2 (refined verisimilitude) Refined verisimilitude is defined by $\leqslant^{\mathrm{rv}}=$ $(\preccurlyeq \cdot \sqsubseteq)$, i.e. 


$$
\varphi \leqslant^{\mathrm{rv}} \chi \Leftrightarrow \text { there is a } \psi \text { with } \varphi \preccurlyeq \psi \sqsubseteq \chi
$$

Since both $\sqsubseteq$ and $\preccurlyeq$ are reflexive, they are both subsumed in $\leqslant^{\mathrm{rv}}$. Moreover, this notion of verisimilitude has all desired properties:

Theorem 1 (properties of refined verisimilitude) $\leqslant^{\mathrm{v} v}$ is a preorder that satisfies the content and the likeness condition, contraposition, and commutes with $\vdash$ and $\dashv$.

Proof $\sqsubseteq$ and $\preccurlyeq$ are reflexive, so we have $(\sqsubseteq) \cup(\preccurlyeq) \subseteq\left(\leqslant^{\mathrm{rv}}\right)$. As a consequence, $\leqslant$ rv inherits reflexivity and the content condition from $\sqsubseteq$, and the likeness condition from $\preccurlyeq$. Transitivity, contraposition and commuting with $\vdash$ and $\dashv$ follow from Lemma 2 and the corresponding properties of $\sqsubseteq$ and $\preccurlyeq$.

We give two alternative definitions of $\leqslant^{\mathrm{rv}}$.

Lemma 3 (alternative definitions of refined verisimilitude)

1. $\varphi \leqslant_{t}^{\mathrm{rv}} \psi$ iff there is a t-increasing injection $f:$ atom $(\varphi \wedge \neg \psi \wedge \neg \tau) \rightarrow$ atom $(\psi \wedge \neg \varphi \wedge \neg \tau)$.

2. $\leqslant_{t}^{\mathrm{rv}}$ is the least relation $\leqslant$ satisfying

$$
\begin{gathered}
\left.\varphi \vdash \tau \Rightarrow \varphi \text { is } \leqslant^{\prime} \text {-minimal(i.e. } \varphi \leqslant^{\prime} \psi \text { for all } \psi\right) \\
\text { for all atoms } \alpha, \beta: t(\alpha) \leqslant t(\beta) \Rightarrow \alpha \leqslant \beta \\
\text { if } \varphi \leqslant \leqslant^{\prime} \psi, \varphi^{\prime} \leqslant \leqslant^{\prime} \psi \text { and } \psi \wedge \psi^{\prime} \vdash \perp \text { then } \varphi \vee \varphi^{\prime} \leqslant \leqslant^{\prime} \psi \vee \psi^{\prime}
\end{gathered}
$$

Proof See the Appendix.

\section{Combining Belief Revision and Verisimilitude}

The basic idea of the combination of belief revision and verisimilitude comes down to the following. Revising a knowledge base or theory by replacing unsuccessful content, which is less verisimilar, with content that is more verisimilar, and therefore more successful, must end up with a new theory that is more verisimilar. The problem is of course that in scientific practice the measure of verisimilitude of data and theories is unknown. Intuitively, an important adequacy constraint therefore reads:

If one's preference order is similar to the verisimilitude order,

then the theory after revision must be more verisimilar than

before the revision.

Let us spell out what (12) comes down to in our framework. Suppose that we have an objective similarity $t$ and a subjective preference $p: t$ is the basis for the truth $\tau=$ form $(t)$ and for the verisimilitude relation $\left(\leqslant^{\mathrm{rv}}\right)=\left(\leqslant_{t}^{\mathrm{rv}}\right)$, while $p$ is subjected to revision with $\varphi$. Now the question is: when is the revision form $(p * \varphi)$ more verisimilar than form $(p)$, i.e. when do we have 


$$
\operatorname{form}(p * \varphi) \leqslant t_{t}^{\mathrm{rv}} \text { form }(p)
$$

In other words: when is the revision $p * \varphi$ a step in the right direction? The conditions for (13) spell out the similarity condition of (12). By Lemma 3, these conditions come down to the existence of a $t$-increasing injective function $f$ with

$$
\begin{aligned}
\operatorname{dom}(f) & =\operatorname{atom}(\operatorname{form}(p * \varphi) \wedge \neg \text { form }(p) \wedge \neg \tau) \\
& =\{\alpha \mid \alpha \vdash \varphi \& p(\alpha)=p(\varphi)>0 \& t(\alpha)>0\} \\
\operatorname{rg}(f) & =\operatorname{atom}(\neg \text { form }(p * \varphi) \wedge \text { form }(p) \wedge \neg \tau) \\
& =\{\alpha \mid p(\alpha)=0 \& t(\alpha)>0 \&(\alpha \nvdash \varphi \text { or } p(\varphi)>0)\}
\end{aligned}
$$

When the revision is conservative (i.e. form $(p) \wedge \varphi \nvdash \perp$ and $p(\varphi)=0$ ), then $\operatorname{dom}(f)=\emptyset$ and (13) is obvious. Let us consider the three more interesting cases of Popperian revision where $p(\varphi)>0$.

1. If theory form $(p)$ and evidence $\varphi$ are false (i.e. $\tau \vdash \neg$ form $(p)$ and $\tau \vdash \neg \varphi$ ), then according to Lemma 3 the similarity condition of (12) simply reduces to the existence of a $t$-increasing injection $f: \operatorname{atom}($ form $(p * \varphi)) \rightarrow$ atom $($ form $(p))$.

2. If theory form $(p)$ is false but evidence $\varphi$ is true (i.e. $\tau \vdash \neg$ form $(p)$ and $\tau \vdash \varphi$ ), then according to Lemma 3 the similarity condition of (12) reduces to the existence of a $t$-increasing injection $f:$ atom $($ form $(p * \varphi) \wedge \neg \tau) \rightarrow$ atom $($ form $(p))$.

3. If, on the contrary, theory form $(p)$ is true and evidence $\varphi$ is false (i.e. $\tau \vdash$ form $(p)$ and $\tau \vdash \neg \varphi)$, then according to Lemma 3 the similarity condition of (12) equals to the existence of a $t$-increasing injection $f:$ atom $($ form $(p * \varphi)) \rightarrow$ atom $($ form $(p) \wedge \neg \tau)$.

The previous cases spell out the exact sense in which one's preference order should be similar, in the sense of having resemblance or likeness, to the verisimilitude order to guarantee improvement of verisimilitude after revision. Perhaps the second case fits best the situation of actual scientific research, where the theory used is probably false but the observations may be (approximately) true due to careful measurements. To illustrate this case let us reconsider Hansson's example of Sect. 1.

Example 2 (Hansson's example reconsidered) In Hansson's example the theory (cq. body of knowledge) form $(p)=P_{0} \wedge \neg P_{1}$ was confronted with new evidence $\varphi=P_{0} \leftrightarrow P_{1}$ under the assumption that the truth $\tau$ equals the atom $P_{0} \wedge P_{1}$. Additionally, the various preference orders $p_{1}, p_{2}, p_{3}$ corresponding with (i), (ii), (iii) of Example 1, are defined by

\begin{tabular}{lllll}
\hline & $P_{0} \wedge P_{1}$ & $P_{0} \wedge \neg P_{1}$ & $\neg P_{0} \wedge P_{1}$ & $\neg P_{0} \wedge \neg P_{1}$ \\
\hline $\mathrm{t}$ & 0 & 1 & 1 & 2 \\
$p_{1}$ & 1 & 0 & 2 or 3 & 2 \\
$p_{2}$ & 2 & 0 & 2 or 3 & 1 \\
$p_{3}$ & 1 & 0 & 2 or 3 & 1 \\
$\varphi$ & 0 & 1 & 1 & 0 \\
\hline
\end{tabular}


It follows that form $\left(p_{i}\right)=P_{0} \wedge \neg P_{1}$ for $i=1,2,3$, and form $\left(p_{1} * \varphi\right)=P_{0} \wedge$ $P_{1}$, form $\left(p_{2} * \varphi\right)=\neg P_{0} \wedge \neg P_{1}$ and finally form $\left(p_{3} * \varphi\right)=P_{0} \leftrightarrow P_{1}$. The injection $f:$ atom (form $\left.\left(p_{1} * \varphi\right) \wedge \neg \tau\right) \rightarrow$ atom(form $\left.\left(p_{1}\right)\right)$ is an empty function and hence $t$ increasing, so form $\left(p_{1} * \varphi\right) \leqslant{ }^{\mathrm{rv}}$ form $\left(p_{1}\right)$, i.e. the revision is at least as verisimilar as the original theory. For $i=2,3$, there is no $t$-increasing injection $f$ : atom $\left(\right.$ form $\left(p_{i} *\right.$ $\varphi) \wedge \neg \tau) \rightarrow$ atom $\left(\right.$ form $\left.\left(p_{i}\right)\right)$, since $t\left(\neg P_{0} \wedge \neg P_{1}\right)=2>1=t\left(P_{0} \wedge \neg P_{1}\right)$.

Returning to the start of the present section, one might ask what can be said more in general about conditions for (13) in terms of the domain and the range of the injective function $f$ ? The injectivity of $f$ can be guaranteed by e.g.

$$
\text { \#atom }(\varphi \wedge \neg \tau) \leqslant \text { \#atom }(\text { form }(p) \wedge \neg \tau) \text {, }
$$

and the $t$-increasingness of $f$ follows from e.g.

$$
\forall \alpha(\alpha \vdash \varphi \wedge \neg \tau \Rightarrow t(\alpha) \leqslant t(\text { form }(p) \wedge \neg \tau))
$$

We may summarize the conjunction of (14) and (15) in the following way.

The revision $p \longmapsto p * \varphi$ is a step in the good direction (form $(p * \varphi) \leqslant{ }_{t}^{\mathrm{rv}}$ form $(p)$ ) when the number of $\neg \tau$-models of $\varphi$ is at most the number of $\neg \tau$-models of form $(p)$, and they are $t$-preferred to them.

We observe that the conclusion holds trivially when $\varphi$ has no models of $\neg \tau$, i.e. when $\varphi \vdash \tau$. But when $\varphi \nvdash \tau$, (13) requires that there are only few $\neg \tau$-models for $\varphi$ and that they are preferred to the $\neg \tau$-models of form $(p)$.

With similar reasoning, we have the following.

The revision $p \longmapsto p * \varphi$ is a step in the wrong direction (form $(p)<_{t}^{\mathrm{rv}}$ form $(p * \varphi))$ when the number of models of form $(p)$ is smaller than the number of $\neg \tau$-models of $\varphi$, and they are $t$-preferred to them.

Finally, we look at the situation that $t=p$, so form $(p)=\tau$. In that case form $(t *$ $\varphi) \leqslant{ }_{t}^{\mathrm{rv}} \tau$ iff the revision is conservative, i.e. $\varphi \wedge \tau \forall \perp$ and hence form $(t * \varphi)=$ $\tau \wedge \varphi$.

\section{Discussion and Conclusions}

In the foregoing we have seen that (iterated) AGM belief revision and refined verisimilitude fit reasonably well together. What does this mean philosophically? From the viewpoint of belief revision this means that the epistemic entrenchment approach is in a good position to be extended with considerations of truth and even verisimilitude. For refined verisimilitude our results imply that it has found an established and well-studied answer to its accompanying epistemic question. Our approach even puts forward a formal underpinning of the intuitively plausible idea that the better theory has the better consequences and doing so it successfully accepted Laudan's challenge. What is the reason for the two approaches to fit so well in contrast to other attempts to combine verisimilitude and belief revision? The answer to this question is connected to the way refined verisimilitude and AGM 
belief revision are constructed. Content definitions of verisimilitude are defined in terms of logical content and truth-value and the same holds true AGM-belief revisions. In both approaches strength prevails over the ordering of worlds. The described match between belief revision and refined verisimilitude provides an important external argument in favor of the refined content approach. ${ }^{2}$

Future research in this direction has to address at least the following three issues. Firstly we would like to explore more extensively the differences between the four rules of iterated belief revision (see Sect. 5), and the different ways in which they approach the truth. Secondly we would like to investigate whether our framework can be extended to languages with infinitely many formulae. Thirdly, we would like to address the question of the completeness of the empirical truth (in other words: whether $\tau$ is an atom or not). We observe that our results do not require the truth to be complete. If the truth is incomplete, some sentences do not acquire a definite truth-value. We would like to find out whether this lack of truth-value of some sentences together with the application of iterative belief revision to refined verisimilitude could assist in deciding between the appropriateness of languages for some area of research.

Open Access This article is distributed under the terms of the Creative Commons Attribution Noncommercial License which permits any noncommercial use, distribution, and reproduction in any medium, provided the original author(s) and source are credited.

\section{Appendix}

\section{Proofs}

In the proofs, we use the notation $f[\varphi]$ to abbreviate $\bigvee f[\operatorname{atom}(\varphi)]$, i.e. $\bigvee\{f(\alpha) \mid \alpha \in \operatorname{atom}(\varphi)\}$; and $f^{-1}[\varphi]$ to denote $\bigvee\{\alpha \mid f(\alpha) \in$ atom $(\varphi)\}$.

Lemma $1 \sqsubseteq$ and $\preccurlyeq$ are preorders, satisfy contraposition and commute with $\vdash$ and $\dashv$. Moreover, $\sqsubseteq$ satisfies the content condition and $\preccurlyeq$ the likeness condition, but not the other way round.

Proof It is easily checked that $\sqsubseteq$ is reflexive, transitive, satisfies the content condition and contraposition. To see that $\sqsubseteq$ and $\vdash$ commute, observe that if $\varphi \sqsubseteq \psi \vdash$ $\chi$, then $\varphi \vdash(\psi \vee \tau) \sqsubseteq \chi$, and if $\varphi \vdash \theta \sqsubseteq \chi$ then $\varphi \sqsubseteq(\theta \wedge \neg \tau) \vdash \chi$. Finally $\sqsubseteq$ and $\dashv$ commute, for both $\sqsubseteq$. $\dashv$ and $\dashv \cdot \sqsubseteq$ are the full relation on FORM. The likeness condition fails for $\sqsubseteq$ : if $\alpha \neq \beta$ and $\min (t)<t(\alpha) \leqslant t(\beta)$ then $\alpha \sqsubseteq \beta$.

For $\preccurlyeq$, reflexivity is easy (with the empty function as witness), and transitivity follows straightforwardly with definition (8) and composition of the witnessing functions. For contraposition, we use definition (7), observing that a witness for $\varphi \preccurlyeq \psi$ is also a witness for $\neg \psi \preccurlyeq \neg \varphi$. A counterexample for the content condition for $\preccurlyeq$ is provided by $\alpha, \beta$ with $\alpha \neq \beta$, for then $\alpha \vdash(\alpha \vee \beta) \vee \tau$, but $\alpha \nprec(\alpha \vee \beta)$.

\footnotetext{
${ }^{2}$ For more information about the distinction between likeness and content verisimilitude definitions, see Zwart and Franssen (2007).
} 
We now show that $(\preccurlyeq \cdot \vdash)=(\vdash \preccurlyeq)$, and start with the $\subseteq$ part. Assume $\varphi \preccurlyeq \psi \vdash \chi$, then there is a $t$-increasing bijection $f: \operatorname{atom}(\varphi \wedge \neg \psi) \rightarrow$ atom $(\psi \wedge \neg \varphi)$. Define

$$
\begin{aligned}
& \theta=\varphi \vee(\chi \wedge \neg f[\varphi \wedge \neg \chi]) \\
& g=f\lceil\operatorname{atom}(\varphi \wedge \neg \chi)
\end{aligned}
$$

then one easily checks that $\varphi \vdash \theta$ and $g$ is a $t$-increasing bijection $g$ : atom $(\theta \wedge$ $\neg \chi) \rightarrow$ atom $(\chi \wedge \neg \theta)$, so $\theta \preccurlyeq \chi$, which proves the $\subseteq$ part.

For the $\supseteq$ part, we reason as follows. Assume $\varphi \vdash \psi \preccurlyeq \chi$, then via contraposition $\neg \chi \preccurlyeq \neg \psi \vdash \neg \varphi$, so (using the property $(\preccurlyeq \cdot \vdash) \subseteq(\vdash \cdot \preccurlyeq)$ that we just proved) there is a $\theta$ with $\neg \chi \vdash \theta \preccurlyeq \neg \varphi$, and via contraposition we get $\varphi \preccurlyeq \neg \theta \vdash \chi$. This yield the $\supseteq$ part, so we have proved that $\preccurlyeq$ and $\vdash$ commute.

Finally we show that $(\preccurlyeq \cdot \dashv)=(\dashv \preccurlyeq)$, and begin again with the $\subseteq$ part. Assume $\varphi \preccurlyeq \psi \dashv \chi$, then $\chi \vdash \psi$ and there is a $t$-increasing bijection $f:$ atom $(\varphi \wedge \neg \psi) \rightarrow$ atom $(\psi \wedge \neg \varphi)$. Define

$$
\begin{aligned}
& \theta=(\varphi \wedge \chi) \vee f^{-1}[\chi \wedge \neg \varphi] \\
& g=f\left\lceil\operatorname{atom}\left(f^{-1}[\chi \wedge \neg \varphi]\right)\right.
\end{aligned}
$$

then $\theta \vdash \varphi$ and $g$ is a $t$-increasing bijection $g:$ atom $(\theta \wedge \neg \chi) \rightarrow$ atom $(\chi \wedge \neg \theta)$, so $\theta \preccurlyeq \chi$, and we have proved the $\subseteq$ part. The other direction is now proved with help of contraposition, as above.

Lemma $2 \sqsubseteq$ and $\preccurlyeq$ commute, i.e. $(\sqsubseteq \cdot \preccurlyeq)=(\preccurlyeq \cdot \sqsubseteq)$.

Proof For increasing bijective functions $f$, we have:

$$
\begin{gathered}
f\left[\varphi^{\prime}\right] \vdash \bigvee \operatorname{rg}(f) \\
f\left[\varphi^{\prime} \wedge \varphi^{\prime \prime}\right] \equiv f\left[\varphi^{\prime}\right] \wedge f\left[\varphi^{\prime \prime}\right] \\
f[\neg \tau] \vdash \neg \tau
\end{gathered}
$$

The first property is evident, the second follows from the injectivity of $f$, and the third uses that $f$ is increasing and that, for all atoms $\alpha \in \operatorname{atom}(\tau)$ and $\beta \notin$ atom $(\tau), t(\alpha)<t(\beta)$. We shall use these properties in the proof.

Now we demonstrate $(\sqsubseteq \preccurlyeq) \subseteq(\preccurlyeq \cdot \sqsubseteq)$. So assume $\varphi \sqsubseteq \psi \preccurlyeq \chi$, i.e. $\varphi \vdash \psi \vee \tau$ and there is an increasing bijection $f: \operatorname{atom}(\psi \wedge \neg \chi) \rightarrow$ atom $(\chi \wedge \neg \psi)$. Define

$$
\begin{aligned}
\varphi_{1} & =\varphi \wedge \neg \chi \wedge \neg \tau \\
\varphi_{2} & =\varphi \wedge(\chi \vee \tau) \\
\theta & =f\left[\varphi_{1}\right] \vee \varphi_{2} \\
g & =f\left\lceil\operatorname{atom}\left(\varphi_{1}\right)\right.
\end{aligned}
$$

First we establish $\theta \sqsubseteq \chi$, i.e. $\theta \vdash \chi \vee \tau$ : this follows from the definition of $\theta$ and the fact that $f\left[\varphi_{1}\right] \vdash \bigvee \operatorname{rg}(f) \vdash \chi$.

Now we shall show that $g$ witnesses $\varphi \preccurlyeq \theta$. It follows directly from the definition of $g$ that it is increasing and injective, so it remains to be shown that $\operatorname{dom}(g)=$ atom $(\varphi \wedge \neg \theta)$ and $\operatorname{rg}(g)=\operatorname{atom}(\theta \wedge \neg \varphi)$. Using $\varphi \vdash \psi \vee \tau$, we observe that atom $\left(\varphi_{1}\right) \subseteq \operatorname{atom}(\psi \wedge \neg \chi)=\operatorname{dom}(f)$, so $\operatorname{dom}(g)=$ atom $\left(\varphi_{1}\right)$. Furthermore, it is 
obvious that $\varphi_{1}, \varphi_{2}$ is a partition of $\varphi$, i.e. $\varphi_{1} \wedge \varphi_{2} \vdash \perp$ and $\varphi_{1} \vee \varphi_{2} \equiv \varphi$. Moreover, we have $f\left[\varphi_{1}\right] \wedge \varphi \vdash \perp$ (using $\operatorname{rg}(f) \subseteq$ atom $(\neg \psi), f[\neg \tau] \vdash \neg \tau$ and $\varphi \vdash \psi \vee \tau)$. So $\varphi_{1}, \varphi_{2}$ and $f\left[\varphi_{1}\right]$ are mutually disjoint. Hence $\varphi \wedge \neg \theta \equiv \varphi_{1}$ and $\theta \wedge \neg \varphi \equiv f\left[\varphi_{1}\right]$. Using $\varphi \vdash \psi \vee \tau$ again, we observe that atom $\left(\varphi_{1}\right) \subseteq$ atom $(\psi \wedge$ $\neg \chi)=\operatorname{dom}(f)$, so we obtain $\operatorname{dom}(g)=\operatorname{atom}\left(\varphi_{1}\right)=\varphi \wedge \neg \theta$. Furthermore $\operatorname{rg}(g)=\operatorname{atom}\left(f\left[\varphi_{1}\right]\right)=\operatorname{atom}(\theta \wedge \neg \varphi)$.

So we have established $(\sqsubseteq \cdot \preccurlyeq) \subseteq(\preccurlyeq \cdot \sqsubseteq)$. For the other way round, we argue as in the proof of Lemma 1 . Assume $\varphi \preccurlyeq \psi \sqsubseteq \chi$, then by contraposition $\neg \chi \sqsubseteq \neg \psi \preccurlyeq \neg \varphi$, and by the result just proved we obtain a $\theta$ with $\neg \chi \preccurlyeq \theta \sqsubseteq \neg \varphi$, so again by contraposition $\varphi \sqsubseteq \neg \theta \preccurlyeq \chi$. This demonstrates $(\preccurlyeq \cdot \sqsubseteq) \subseteq(\sqsubseteq \cdot \preccurlyeq)$, which ends the proof.

\section{Lemma 3}

1. $\varphi \leqslant{ }^{\mathrm{r}} \psi$ iff there is an increasing injection $f:$ atom $(\varphi \wedge \neg \psi \wedge \neg \tau) \rightarrow$ atom $(\psi \wedge \neg \varphi \wedge \neg \tau)$.

$2 . \quad \leqslant$ iv the least relation $\leqslant$ satisfying

$$
\begin{gathered}
\left.\varphi \vdash \tau \Rightarrow \varphi \text { is } \leqslant^{\prime} \text {-minimal (i.e. } \varphi \leqslant^{\prime} \psi \text { for all } \psi\right) \\
\text { for all atoms } \alpha, \beta: t(\alpha) \leqslant t(\beta) \Rightarrow \alpha \leqslant \leqslant^{\prime} \beta \\
\text { if } \varphi \leqslant \leqslant^{\prime} \psi, \varphi^{\prime} \leqslant \text { ' } \psi \text { and } \psi \wedge \psi^{\prime} \vdash \perp \text { then } \varphi \vee \varphi^{\prime} \leqslant{ }^{\prime} \psi \vee \psi^{\prime}
\end{gathered}
$$

\section{Proof}

1. $\Rightarrow$ : If $\varphi \leqslant^{\mathrm{rv}} \psi$ then $\varphi \sqsubseteq \theta \preccurlyeq \psi$ for some $\theta$, i.e. $\varphi \vdash \theta \vee \tau$ and there is an increasing bijection $g$ : atom $(\theta \wedge \neg \psi) \rightarrow$ atom $(\psi \wedge \neg \theta)$. Define $f:=g$ |atom $(\varphi \wedge \neg \psi \wedge$ $\neg \tau)$. By $\varphi \vdash \theta \vee \tau$ we have $\varphi \wedge \neg \psi \wedge \neg \tau \vdash \theta \wedge \neg \psi$, so $\operatorname{dom}(g)=\operatorname{atom}(\varphi \wedge$ $\neg \psi \wedge \neg \tau)$; and $\operatorname{rg}(g) \subseteq$ atom $(\psi \wedge \neg \varphi \wedge \neg \tau)$ follows from $\varphi \vdash \theta \vee \tau$ and $f[\neg \tau] \vdash \neg \tau$ (which we demonstrated in the proof of Lemma 2).

$\Leftarrow$ : Let $f:$ atom $(\varphi \wedge \neg \psi \wedge \neg \tau) \rightarrow$ atom $(\psi \wedge \neg \varphi \wedge \neg \tau)$ be an increasing injection. Define $\theta:=\bigvee \operatorname{rg}(f) \vee(\varphi \wedge(\psi \vee \tau))$, then $\varphi \wedge \neg \theta \equiv \varphi \wedge \neg \psi \wedge \neg \tau$ and $\theta \wedge \neg \varphi \equiv \bigvee \operatorname{rg}(f)$, so $f:$ atom $(\varphi \wedge \neg \theta) \rightarrow$ atom $(\theta \wedge \neg \varphi)$ is bijective, so $\varphi \preccurlyeq \theta$. Also $\theta \vdash \psi \vee \tau$, so $\theta \sqsubseteq \psi$. By Lemma 2 we now have $\varphi \leqslant^{\mathrm{rv}} \psi$.

2. First we show that $\leqslant^{\mathrm{rv}}$ satisfies the three conditions, using the characterization of $\leqslant^{\mathrm{rv}}$ proved in the first part of this lemma. If $\varphi \vdash \tau$, then atom $(\varphi \wedge \neg \psi \wedge$ $\neg \tau)=\emptyset$ for all $\psi$, so the empty function witnesses $\varphi \leqslant{ }^{\mathrm{r}} \psi$ for all $\psi$, i.e. (9). If $t(\alpha) \leqslant t(\beta)$, then either $f=\{(\alpha, \beta)\}$ (if $\alpha \forall \beta \vee \tau$ ) or $f=\emptyset$ witnesses that $\alpha \leqslant{ }^{\mathrm{rv}} \beta$, establishing (10). Finally, if $\varphi \leqslant{ }^{\mathrm{rv}} \psi$ and $\varphi^{\prime} \leqslant{ }^{\mathrm{rv}} \psi^{\prime}$ then we have increasing injections $f: \operatorname{atom}(\varphi \wedge \neg \psi \wedge \neg \tau) \rightarrow \operatorname{atom}(\psi \wedge \neg \varphi \wedge \neg \tau)$ and $f^{\prime}: \operatorname{atom}\left(\varphi^{\prime} \wedge\right.$ $\left.\neg \psi^{\prime} \wedge \neg \tau\right) \rightarrow$ atom $\left(\psi^{\prime} \wedge \neg \varphi^{\prime} \wedge \neg \tau\right)$. Define $g=f \cup\left(f^{\prime} \uparrow\left(\operatorname{dom}\left(f^{\prime}\right)-\operatorname{dom}(f)\right)\right)$. Injectivity of $g$ follows from $\psi \wedge \psi^{\prime} \vdash \perp$, and we see that $g$ witnesses that $\varphi \vee \varphi^{\prime} \leqslant{ }^{\mathrm{r} \vee} \psi \vee \psi^{\prime}$, i.e. (11). It remains to show that $\leqslant^{\mathrm{rv}}$ is the least relation that satisfies the thee conditions. So let $\leqslant$ ' satisfy the conditions: we shall show that $\leqslant^{\mathrm{rv}}$ is a subrelation of $\leqslant^{\prime}$, i.e. $\varphi \leqslant^{\prime} \psi \Rightarrow \varphi \leqslant^{\mathrm{rv}} \psi$ for all $\varphi, \psi$. Assume that 
$\varphi \leqslant{ }^{\mathrm{rv}} \psi$, then by the first part of this lemma there is an increasing injection $f:$ atom $(\varphi \wedge \neg \psi \wedge \neg \tau) \rightarrow$ atom $(\psi \wedge \neg \varphi \wedge \neg \tau)$. By (11), $\varphi \leqslant \leqslant^{\prime} \psi$ follows from

$$
\begin{aligned}
& \varphi \wedge \psi \leqslant^{\prime} \varphi \wedge \psi \\
& \varphi \wedge \neg \psi \wedge \neg \tau \leqslant \leqslant^{\prime} \bigvee \operatorname{rg}(f) \\
& \varphi \wedge \tau \leqslant^{\prime} \psi \wedge \neg \varphi \wedge \neg \bigvee \operatorname{rg}(f)
\end{aligned}
$$

for $\quad \varphi \equiv(\varphi \wedge \psi) \vee(\varphi \wedge \neg \psi \wedge \neg \tau) \vee(\varphi \wedge \tau), \psi \equiv(\varphi \wedge \psi) \vee(\vee \operatorname{rg}(f)) \vee(\psi \wedge$ $\neg \varphi \wedge \neg \bigvee \operatorname{rg}(f))$ (use that $\bigvee \operatorname{rg}(f) \vdash \psi$ ); moreover, $\varphi \wedge \psi, \bigvee \operatorname{rg}(f)$ and $\psi \wedge \neg \varphi \wedge$ $\neg \bigvee \operatorname{rg}(f)$ are mutually disjoint (use that $\bigvee \mathrm{rg}(f) \vdash \neg \varphi$ ).

We set out to prove these three inequalities. By (10) we have $\alpha \leqslant^{\prime} \alpha$ for all atoms $\alpha$, and $\beta \leqslant^{\prime} f(\beta)$ for all atoms $\beta \in \operatorname{dom}(f)$ (for $f$ is $t$-increasing). By repeated application of (11), we get $\varphi \wedge \psi \leqslant \leqslant^{\prime} \varphi \wedge \psi$ and $\varphi \wedge \neg \psi \wedge \neg \tau=\bigvee \operatorname{dom}(f) \leqslant^{\prime} \bigvee \operatorname{rg}(f)$ Finally, $\varphi \wedge \tau \leqslant \leqslant^{\prime} \psi \wedge \neg \varphi \wedge \neg \bigvee \operatorname{rg}(f)$ follows from (9). We conclude that $\varphi \leqslant^{\prime} \psi$, so we have proved that $\leqslant^{\mathrm{rv}}$ is a subrelation of $\leqslant^{\prime}$.

\section{References}

Alchourrón, C. E., Gärdenfors, P., \& Makinson, D. (1985). On the logic of theory change: Partial meet functions for contraction and revision. Journal of Symbolic Logic, 50, 510-530.

Darwiche, A., \& Pearl, J. (1997). On the logic of iterated belief revision. Artificial Intelligence, 89(1-2), $1-29$.

Duhem, P. (1954). The aim and structure of physical theory. Princeton University Press, Princeton

Grove, A. (1988). Two modellings for theory change. Journal of Philosophical Logic, 17, 157-170.

Kuipers, T. A. F. (2000). From instrumentalism to constructive realism: On some relations between confirmation, empirical progress, and truth approximation. Princeton: Springer.

Laudan L. (1981). A confutation of convergent realism. Philosophy of Science, 48, 19-49.

Miller, D. (1974). Popper's qualitative theory of verisimilitude. British Journal for the Philosophy of Science, 25, 166-177.

Miller, D. (1978). On distance from the truth as a true distance. In J. Hintikka, I. Niiniluoto, \& E. Saarinen (Eds.) Essays on mathematical and philosophical logic (pp. 15-26). Dordrecht: Reidel.

Nayak, A. C. (1994). Iterated belief change based on epistemic entrenchment. Erkenntnis, 41, 353-390.

Niiniluoto, I. (1987). Truthlikeness. Dordrecht, The Netherlands: Reidel.

Popper, K. (1963). Conjectures and refutations. London: Routledge and Kegan Paul.

Quine, W. V., \& Ullian, J. S. (1978). The web of belief (2nd ed). New York: McGraw-Hill Humanities/ Social Sciences/Languages.

Zwart, S. D. (2001). Refined verisimilitude. Dordrecht, The Netherlands: Kluwer Academic Press.

Zwart, S. D., \& Franssen, M. (2007). An impossibility theorem for verisimilitude. Synthese, 158, 75-82. 